\title{
Rhinovirus infections and immunisation induce cross-serotype reactive antibodies to VP1
}

\author{
Gary R. McLean a,*,1,2, Ross P. Walton ${ }^{\mathrm{a}, 2}$, Shweta Shetty ${ }^{\mathrm{a}}$, Nasren Paktiawal ${ }^{\mathrm{a}}$, Tatiana Kebadze ${ }^{\mathrm{a}}$, \\ Leila Gogsadze ${ }^{\mathrm{a}}$, Katarzyna Niespodziana ${ }^{\mathrm{b}, 2}$, Rudolf Valenta ${ }^{\mathrm{b}, \mathrm{c}, 2}$, Nathan W. Bartlett ${ }^{\mathrm{a}, 2}$, \\ Sebastian L. Johnston ${ }^{\mathrm{a}, *, 1,2}$

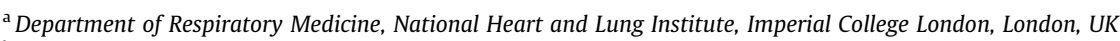 \\ ${ }^{\mathrm{b}}$ Division of Immunopathology, Dept. of Pathophysiology and Allergy Research, Center for Pathophysiology, Infectiology and Immunology, Medical University of Vienna, \\ Vienna, Austria \\ ${ }^{\mathrm{c}}$ Christian Doppler Laboratory for Allergy Research, Medical University of Vienna, Vienna, Austria
}

\section{A R T I C L E I N F O}

\section{Article history:}

Received 16 May 2012

Revised 14 June 2012

Accepted 18 June 2012

Available online 26 June 2012

\section{Keywords:}

Antibodies

Rhinovirus

Vaccine

Mouse model

\begin{abstract}
A B S T R A C T
Rhinoviruses (RVs) are ubiquitous human respiratory viruses, the major cause of common colds, acute exacerbations of asthma and other respiratory diseases. The development of antibodies to RV following primary infection is poorly understood and there is currently no RV vaccine available. We therefore used mouse models of intranasal RV infection and immunisation to determine the induction, magnitude and specificity of antibody responses. Strong cross-serotype RV-specific IgG responses in serum and bronchoalveolar lavage were induced towards the RV capsid protein VP1. IgA responses were weaker, requiring two infections to generate detectable RV-specific binding. Similarly two or more RV infections were necessary to induce neutralising antibodies. Immunisation strategies boosted homotypic as well as inducing cross-serotype neutralising IgG responses. We conclude that VP1 based antigens combined with adjuvants may permit successful antibody-mediated vaccine design and development.
\end{abstract}

(c) 2012 Published by Elsevier B.V.

\section{Introduction}

RV's are ubiquitous human pathogens of the respiratory tract. They are the major cause of common colds and acute exacerbations of airway diseases such as asthma and chronic obstructive pulmonary disease (COPD), are responsible for approximately 50\% of virus-induced respiratory infections and half to two-thirds of virus-induced asthma exacerbations (Greenberg, 2007; Johnston et al., 1995). RV belongs to the single-stranded positive sense RNA virus Picornaviridae family. The RNA genome is surrounded by an icosahedral protein capsid comprising 60 copies of 4 structural proteins, the surface exposed VP1, VP2, VP3 and the buried VP4 (Rossmann et al., 1985). One hundred distinct serotypes of RV numbered 1-100 have been well characterised (Rhinoviruses: a numbering system, 1967; Conant and Hamparian, 1968a,b) and classified based on antibody neutralisation properties with guinea

\footnotetext{
* Corresponding authors. Address: Department of Respiratory Medicine, Imperial College London, Norfolk Place, London W2 1PG, UK. Tel.: +44 207594 3764; fax: +44 2072628913 (S.L. Johnston).

E-mail addresses: g.mclean@imperial.ac.uk (G.R. McLean), s.johnston@imperial.ac.uk (S.L. Johnston).

${ }^{1}$ Current address: School of Human Sciences, London Metropolitan University, London, UK.

2 PreDicta Consortium Member.
}

pig antisera (Schieble et al., 1974). RV can be further grouped based upon receptor usage with approximately $90 \%$ of serotyped strains (major group) using ICAM-1 as the receptor to enter host epithelial cells (Greve et al., 1989) whereas the minor group is known to exploit members of the low-density lipoprotein (LDL) receptor family (Hofer et al., 1994).

In humans, protection against re-infection with RV is associated with high levels of serotype-specific neutralising antibody both in serum and respiratory secretions (Barclay et al., 1989). Vaccination with RV preparations also induces neutralising antibodies in humans with IgA being the dominant antibody found in nasal secretions whereas in serum IgG dominates (Knopf et al., 1970; Perkins et al., 1969). In addition, high levels of RV-binding serum IgA correlates well with total serum neutralising antibody levels, the lack of development of a cold, and reduced viral shedding following experimental RV infection (Barclay and Al-Nakib, 1987; Barclay et al., 1988). The relative contributions of the various human IgG and IgA subclasses to RV neutralisation has not been investigated extensively although RV-binding IgG1, IgG4, and IgA1 levels rise in response to RV infection and neutralising titres are correlated with both IgG1 and IgA1 levels (Carey et al., 1992). In most cases, neutralising antibodies generated to RV following immunisation or infection are highly serotype-specific (Conant and Hamparian, 1968a,b), nevertheless in some cases, antibodies persisting from 
previous encounters with other RV serotypes have been demonstrated to cross-react with closely related RV serotypes and rarely to result in cross-neutralisation (Fox, 1976). Thus, there are limited data from humans supporting the development of vaccine strategies that induce cross-serotype neutralising antibodies against RV. However, the lack of a practical mouse model of RV infection has been a major barrier for vaccine development and the investigation of the fundamentals of RV antibody generation.

Complicating the study of antibody generation following RV infection is the fact that humans experience frequent RV infections throughout life (Turner, 1997) and therefore develop RV-specific antibodies from an early age. Recently a mouse model of RV infection was developed (Bartlett et al., 2008). Intranasal infection of minor (BALB/c) and major group (transgenic BALB/c expressing mouse-human ICAM-1 chimaera) RV induced airway inflammatory responses and viral replication was demonstrated in airway epithelium (Bartlett et al., 2008). The major advantage of using a mouse model of RV infection for our studies is, unlike humans, mice are immunologically naive to RV and the development of the humoral immune responses can be directly studied without interference from cross reactive antibodies. We have now used this model to characterise the induction of humoral immune responses generated after single and multiple i.n. RV infections, studies that are difficult to perform in humans. We determined that antibody responses were predominantly $\operatorname{IgG}$, were directed against the RV capsid protein VP1 and multiple RV infections were required to generate neutralising antibodies. We also report that these responses can be augmented by mucosal $\mathrm{CpG}$, as well as by systemic immunisation with inactivated virus and adjuvant. The latter approach boosted serotype-specific as well cross-serotype neutralising antibody. Our findings demonstrate the utility of the mouse model for investigating anti-RV humoral immunity and for determining the effect of vaccine strategies on the response to RV infection. VP1 based antigens combined with adjuvants have promise as candidate vaccines to boost serotype-specific as well as cross-serotype neutralising antibodies.

\section{Materials and methods}

\subsection{Rhinovirus preparations}

Serotypes RV1B, RV29 and RV16 were produced by culture in HeLa cells as described previously (Bartlett et al., 2008). To investigate virus-specificity of antibody binding, control HeLa lysate was prepared in an identical fashion from uninfected cells. RV preparations were inactivated by exposing them to UV light $(1,200 \mathrm{~mJ} / \mathrm{cm} 2$ for $30 \mathrm{~min}$ ) as previously described (Bartlett et al., 2008). Recombinant VP1 from RV14 and RV89 was expressed and purified as described (Edlmayr et al., 2009, 2011).

\subsection{Animals and experimental protocols}

All animal work was in accordance with Project Licence PPL70/ 6387 , and performed according to regulations outlined by the Home Office, UK, in agreement with the Animals (Scientific Procedures) Act 1986. Female 6-8 week old BALB/c mice were purchased from Harlan and housed under appropriate SPF conditions. RV infections were performed as described previously (Bartlett et al., 2008). Briefly, purified RV $\left(1 \times 10^{6} \mathrm{TCID}_{50}\right)$ in a $50 \mu \mathrm{L}$ volume of PBS was introduced i.n. to groups of 4-6 isoflurane anaesthetised mice.

\subsubsection{Primary infection}

Mice were infected i.n. with RV and sacrificed at time points up to 18 days later.

\subsubsection{Secondary infection}

Mice were infected i.n. with RV, left to resolve the infection for 30 days then re-infected i.n. with homologous or heterologous RV serotypes and sacrificed at time points up to 16 days later. In some experiments, mice were also administered $20 \mu \mathrm{g} \mathrm{CpG}$ oligonucleotides (ODN 1826, Invivogen, Nottingham UK) i.n. 2 days before and at the same time as the first RV infection, left for 30 days and re-infected i.n. with RV with analyses performed 7 days later.

\subsubsection{Multiple infections}

Mice were i.n. infected with RV1B on 5 separate occasions at 14-20 day intervals and sacrificed for endpoint analyses 4 days after the final infection.

\subsubsection{Hyper-immunisation}

Mice were immunised subcutaneously (s.c.) with $0.2 \mathrm{~mL}$ of a solution containing equal volumes of Freund's complete adjuvant (FCA, Sigma Aldrich UK) and RV1B $\left(2 \times 10^{6}\right.$ TCID50). Mice were then further immunised in a similar fashion using Freund's incomplete adjuvant (IFA, Sigma Aldrich UK) and RV1B 4 further times at monthly intervals. Control animals received adjuvant immunisations alone. Mice were infected i.n. with RV1B 1 month after the final immunisation and sacrificed 4 days later.

\subsection{Elisa}

Plates (96-well, Nunc MaxiSorp) were coated overnight at $4{ }^{\circ} \mathrm{C}$ with the purified RV preparations used for i.n. infections and HeLa cell lysates diluted to a protein concentration of $5 \mu \mathrm{g} / \mathrm{mL}$ in PBS or with purified recombinant VP1 proteins diluted to $1 \mu \mathrm{g} / \mathrm{mL}$ in PBS. Plates were blocked for $2 \mathrm{~h}$ at room temperature by adding PBS containing $0.05 \%$ Tween 20 and $5 \%$ milk powder (PBST-milk) before the addition of diluted serum or BAL samples and incubation overnight at $4{ }^{\circ} \mathrm{C}$. Plates were washed with PBST and bound immunoglobulins were detected using biotinylated rat anti-mouse $\operatorname{IgG} 1$, IgG2a, IgG2b or IgA (BD Biosciences UK, Oxford UK) diluted 1:1000 before the addition of streptavidin-peroxidase (Invitrogen, Paisley UK). Plates were developed using tetramethyl benzidine (TMB) solution (Invitrogen, Paisley UK) as substrate and the optical density read using a spectrophotometer at $450 \mathrm{~nm}$ with $570 \mathrm{~nm}$ reference.

\subsection{Immunoblotting}

RV protein preparations were separated by reducing SDS-PAGE, transferred to nitrocellulose membranes, blocked with PBST-milk for $1 \mathrm{~h}$ at room temperature before diluted mouse or guinea pig serum (V-113-501-558, purchased from ATCC) was added and incubated overnight with shaking at $4{ }^{\circ} \mathrm{C}$. Bound serum IgG was analysed using anti-mouse IgG or anti-guinea pig IgG coupled to peroxidase (Sigma Aldrich UK). ECL advance (Amersham Biosciences UK) was added to washed membranes which were then exposed to X-ray film and developed.

\subsection{In vitro $R V$ neutralisation assay}

Mouse serum was serially diluted in a 96-well tissue culture plate in DMEM containing 4\%FCS then $100 \mathrm{TCID}_{50}$ of RV1B or RV16 was added before incubation with shaking at room temperature for 1 h. $2 \times 10^{4}$ HeLa cells were added to each well and the plates incubated at $37^{\circ} \mathrm{C}$ for 3 days before the cytopathic effect (CPE) was inspected microscopically. CPE was measured by staining with crystal violet as described (Edlmayr et al., 2011). Guinea pig antiserum specific for RV1B (V-113-501-558) or RV16 (V105-501-558) were purchased from ATCC and served as a positive control for virus neutralisation. 


\subsection{IgG depletion from antiserum}

Mouse serum was diluted in PBS and allowed to mix with protein $\mathrm{G}$ Sepharose overnight at $4{ }^{\circ} \mathrm{C}$. After centrifugation to remove the Sepharose beads and bound $\operatorname{IgG}$, the unbound fraction containing $\operatorname{IgA}$ was retained and used in ELISA experiments. Depletion of IgG from serum was confirmed by showing loss of binding to RV by ELISA.

\subsection{Flow cytometry}

Leukocytes from BAL and lungs were prepared as described previously (Bartlett et al., 2008). Approximately $5 \times 10^{5}$ cells were suspended in FACS buffer (PBS containing 1\% bovine serum albumin and $0.01 \%$ sodium azide), Fc receptors blocked with rat antimouse CD16/CD32 (BD Biosciences UK, Oxford UK) for 20 min on ice then stained with pacific blue-conjugated rat anti-mouse $\mathrm{CD} 3$ (anti-CD3-PB) and fluorescein isothiocyanate-conjugated rat antimouse CD19 (anti-CD19-FITC) for 30 min on ice. The cells were then washed several times before flow cytometric analysis.

\subsection{B cell ELISpot}

Lung and mediastinal lymph node (MLN) leukocytes were prepared as described previously (Bartlett et al., 2008). Detection of total and RV-specific IgG and IgA production by B cells was performed by ELISpot assay using kits purchased from Mabtech $A B$ (Nacka Strand, Sweden) and following manufacturer instructions.

\section{Results}

\subsection{Intranasal infection of mice with $R V$ rapidly induces cross serotype reactive $\operatorname{Ig} G$ responses}

We investigated the IgG responses generated to RV by BALB/C mice following primary RV infection and re-challenge with homologous (RV1B) or heterologous (RV29) RV serotypes. Mice infected with RV1B or RV29 made RV-specific serum IgG2a and IgG2b that was detectable by ELISA after 8-16 days (upper panels of Fig. 1AD). RV-specificity was confirmed by showing that both IgG2a (Fig. $1 \mathrm{~A}$ and $\mathrm{C}$ ) and IgG2b (Fig. 1B and D) responses in wells coated with RV preparations were significantly greater than responses observed in wells coated with HeLa cell lysates. The use of HeLa lysate as an antigen was important given that RV was grown in these cells and contained HeLa- and serum-derived proteins against which antibodies may be generated. We also observed $\mathrm{RV}$-specific IgG that were serotype cross reactive since both serum IgG2a and IgG2b were generated that bound RV1B after a single RV29 infection (Fig. 1A and B). Following a single RV1B infection only cross reactive serum IgG2b that recognised RV29 was detected (Fig. 1C and D). These data reflect the high degree of capsid protein homology (77\% identity within VP1-VP2-VP3) that exists between RV1B and RV29 which are both type A RV's. Re-challenge of mice by infection with RV1B or RV29 one month after the initial RV1B infection generated increased RV-specific serum IgG responses that was detectable as early as 1 day after the second infection and reached maximal levels after 4-8 days (lower panels of Fig. 1A-D).

\subsection{RV capsid protein VP1 is a major antibody target}

To characterise the IgG binding to RV proteins by serum from RV1B infected mice we performed western blot analyses. Proteins within HeLa lysate, RV1B, RV29 and RV16 preparations were separated by electrophoresis and probed with serum from RV1B in- fected mice or commercially available guinea pig antisera to RV1B. Detection of the bound mouse IgG revealed a virus-specific band of approximately $39 \mathrm{kDa}$ common to RV1B, RV29 and RV16 (Fig. 2A left panel). The $39 \mathrm{kDa}$ band was also observed in each of the three RV preparations when probed with a commercially available guinea pig antisera raised against RV1B (Fig. 2A right panel). It is likely that the $39 \mathrm{kDa}$ band common to each RV preparation is VP1 since it has been shown previously to migrate at this molecular weight (Skern et al., 1987). To confirm the presence of antibodies against VP1 in sera from mice infected twice with RV1B we performed ELISA using purified recombinant VP1 from RV serotypes RV14 and RV89 (Edlmayr et al., 2011) as the antigen since recombinant VP1 from RV1B was unavailable. We observed that mouse IgG2a from RV1B infected animals bound to the more similar VP1 from RV89 (66\% identity between RV1B and RV89 VP1) but not to VP1 from RV14 (43\% identity between RV1B and RV14 VP1) (Fig. 2B upper panels). IgG1 responses were not significant (Fig. 2B lower panels). These data support our notion that antisera from RV infected mice primarily binds to the capsid protein VP1.

\subsection{Multiple RV infections induce IgA responses}

Two RV1B infections were required to generate significant IgA binding to RV1B as detected by ELISA (Fig. 3A). We observed that both bronchoalveolar lavage (BAL) and serum contained detectable RV1B-sepcific IgA 4-8 days after the second RV1B infection whereas a single RV1B infection did not generate significant IgA response to RV1B (Fig. 3A upper panel). RV1B serotype-specific binding was detected in BAL by day 4 post second infection whereas cross reactive BAL IgA responses to RV29 were lower with significant binding not observed until 8 days after two RV1B infections (Fig. 3A lower panel). For IgG isotype antibody responses no RVspecific IgG was found in BAL after a single RV infection but significant binding of IgG2a and IgG1 to RV1B was detected 8-16 days after two infections (Fig. 3B). These data imply that mice infected with RV generate weak mucosal antibody responses or simply they may reflect the fact that lower concentrations of IgA than IgG are found in these samples. To determine if the difficulty in detecting IgA was due to larger amounts of RV-specific IgG blocking access of IgA to antigenic sites we treated serum from RV1B-infected mice with protein $G$ to remove IgG. This reduced the levels of IgG2a that bound RV and permitted the easier detection of RV-specific IgA in serum samples from mice infected multiple times with RV1B (Fig. 3C). These data are consistent with the notion that mice infected with RV generate both systemic and mucosal RV-specific antibody responses and that the higher IgG levels can mask detection of IgA levels.

\subsection{Lung and mediastinal lymph node B lymphocytes produce $R V$ - specific IgG}

To investigate the source of RV-specific antibodies we analysed B lymphocytes and their production of RV-specific antibodies following a single RV infection. We first determined by flow cytometric analyses that $\mathrm{CD}^{-} \mathrm{CD} 19^{+} \mathrm{B}$ lymphocytes were found normally in the lungs and that RV infection induced their appearance in BAL after 4-7 days (SFig. 1A and B). Approximately $8.8 \%$ of BAL lymphocytes were $B$ cells and their numbers were 100 -fold lower than found in lungs (SFig. 1A and 1B). By ELISpot, we were unable to detect RV-specific IgG or IgA production by BAL cells, reflecting the low frequency of $B$ cells found in this anatomical location. However, similar analyses of unfractionated lung (Fig. 4A) and mediastinal lymph node (Fig. 4B) leukocytes showed that RV-specific IgG was produced in a time-dependent manner. Thus, RV-specific IgG production by $\mathrm{B}$ cells was maximal $10-18$ days after a single 

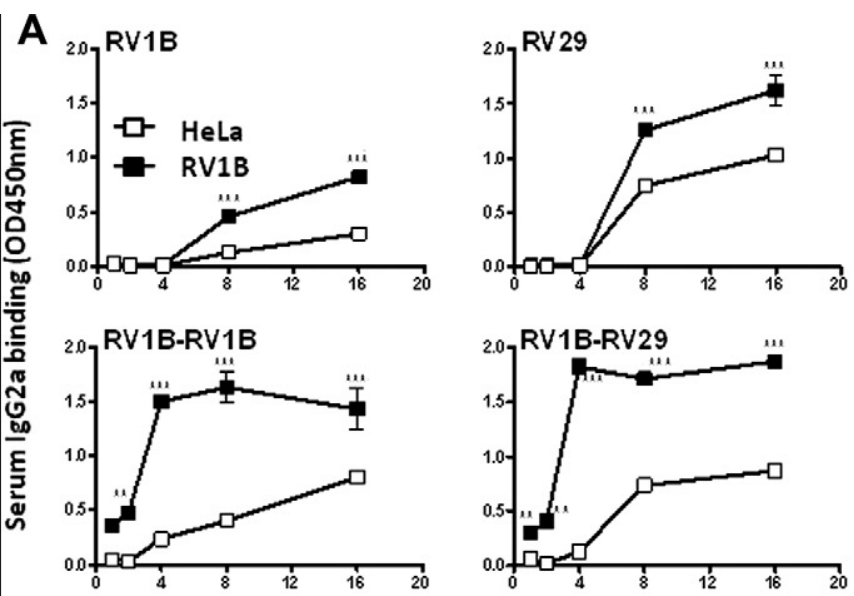

Day after RV infection
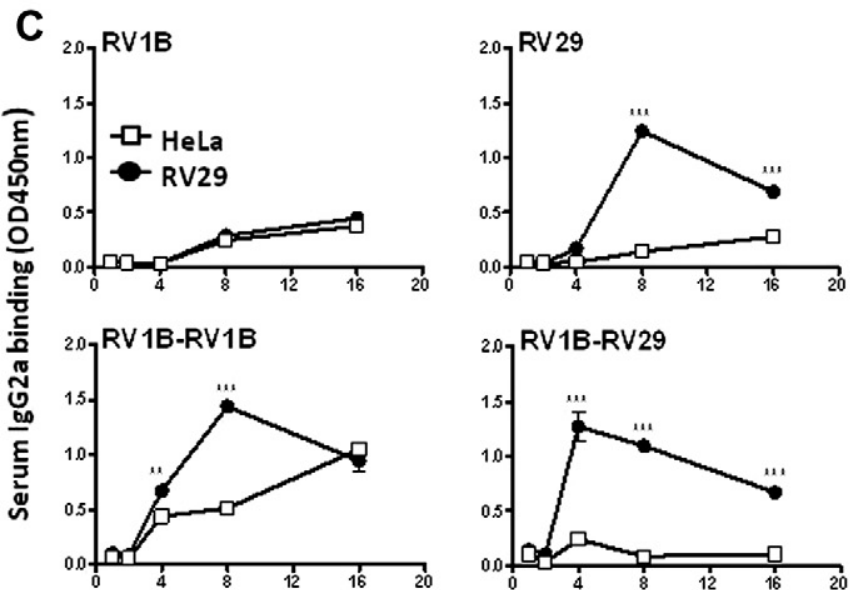

Day after RV infection
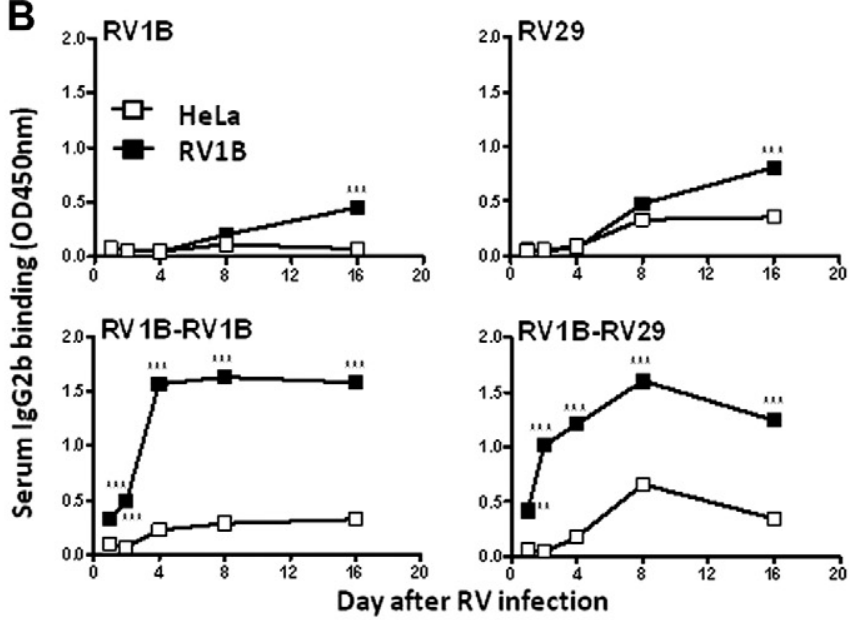

D
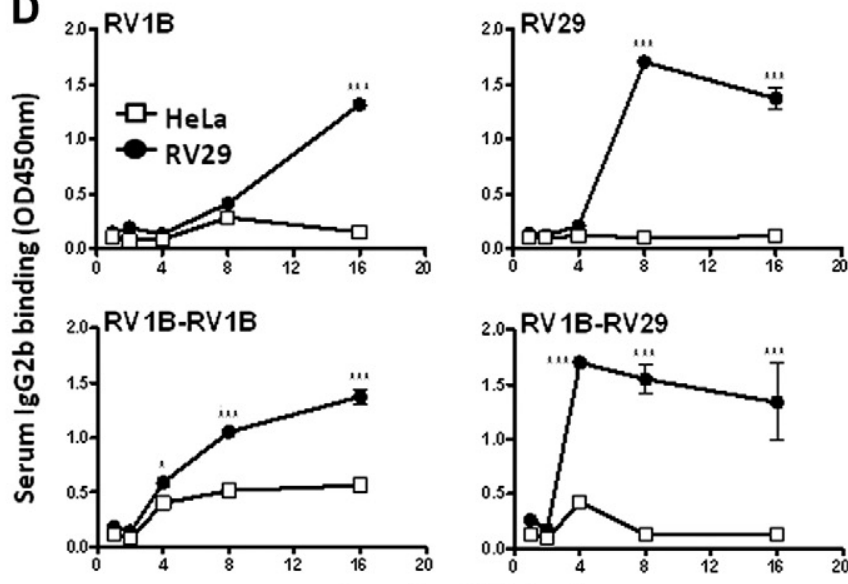

Day after RV infection

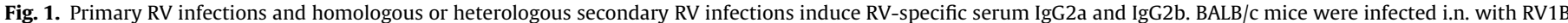

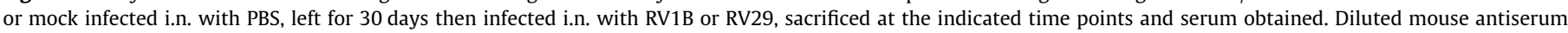

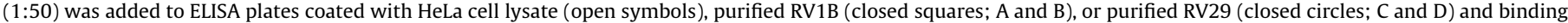
of IgG2a (A and C) and IgG2b (B and D) was assessed. Data are presented as the mean \pm SEM $\left({ }^{*} p<0.05,{ }^{* *} p<0.01,{ }^{* * *} p<0.001\right.$ for HeLa vs. RV).

RV1B infection in both lung and mediastinal lymph node, with a greater frequency of production by cells from the lymph nodes. $\mathrm{RV}$-specific IgA was similarly produced albeit at much lower levels than IgG (Fig. 4A and B), reflecting the masking effect of higher IgG levels or the modest IgA responses observed in serum and BAL.

\subsection{Mucosal CpG administration enhances IgG2a and RV-neutralising antibody responses in response to $R V$ infection}

To promote neutralising antibody generation, we also treated the mice infected with RV with Th1-promoting $\mathrm{CpG}$ oligonucleotides (Fig. 5A). We determined the level of RV1B-specific serum IgG by ELISA following two RV1B i.n. infections 30 days apart, the first infection directly following $\mathrm{CpG}$ or mock i.n. treatment. Antisera from mice that were treated with $\mathrm{CpG}$ and infected with RV1B displayed significantly increased $\operatorname{IgG} 2 \mathrm{a}$ responses but unaltered IgG1 responses to RV1B (Fig. 5B). These data are consistent with the known ability of CpG to promote Th1 immunity (Krieg, 2002) and suggest that memory Th1 immunity to RV1B was encouraged by mucosal $\mathrm{CpG}$ administration at the time of first encounter with RV1B. To determine if the increased Th1-associated IgG2a antibody responses promoted by $\mathrm{CpG}$ resulted in production of neutralising antibodies, we performed in vitro RV neutralisation tests. Antisera from RV-infected and CpG-treated animals contained antibodies that neutralised RV1B infectivity whereas antisera from mice infected twice with RV1B in the absence of $\mathrm{CpG}$ failed to neutralise (Fig. 5C). The neutralising property of the $\mathrm{CpG}$-adjuvanted response was serotype specific as neutralisation of RV16 was not observed (Fig. 5C).

\subsection{Immunisation with $R V$ in Freund's adjuvant generates cross- serotype neutralising IgG}

Having determined that mucosal $\mathrm{CpG}$ administration with live RV1B infection promoted serotype-specific neutralising antibody responses, we investigated whether systemic immunisations might induce cross-serotype neutralising responses (Fig. 6A). Following subcutaneous immunisation with inactivated RV1B in Freund's adjuvant followed by a single intranasal infection with live RV1B we observed high-titre RV-specific serum IgG2a and IgG1 (Fig. 6B). RV-specific serum IgA was not detected following this immunisation protocol. Similarly to i.n. RV1B infections, immunisation with RV1B in adjuvant generated strong serum IgG2a responses to recombinant RV89 VP1 but not to RV14 VP1 (Fig. 6C). 

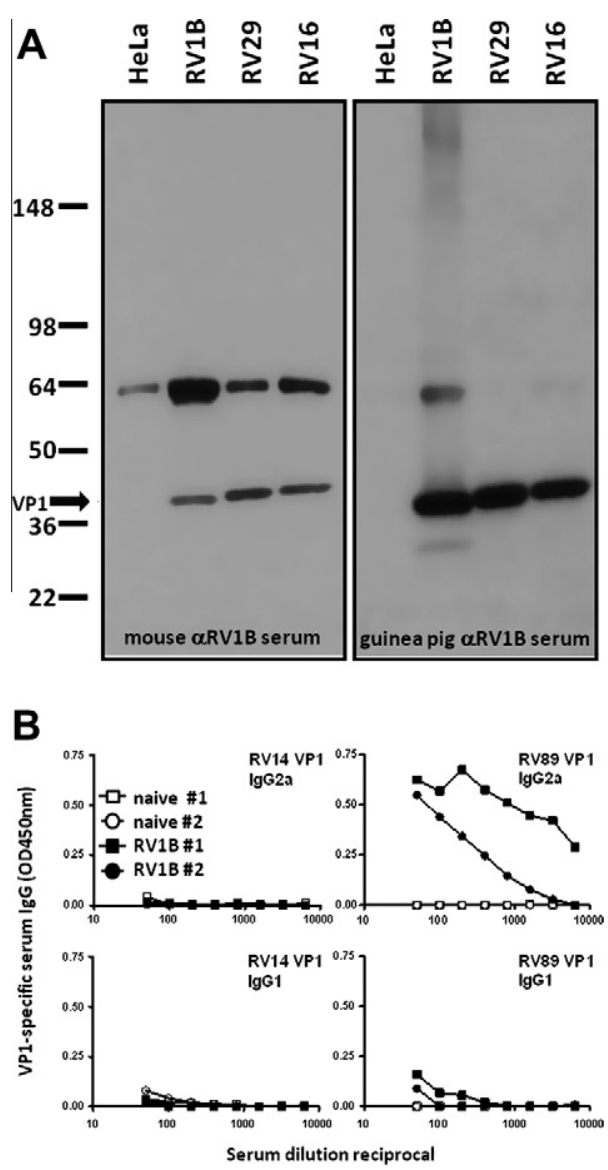

Fig. 2. Antisera from RV infected mice binds to the RV capsid protein VP1. (A) Western blotting was performed to analyse IgG binding to HeLa lysate, RV1B, RV16, and RV29 preparations using diluted antisera (1:500) from mice infected twice with RV1B or diluted guinea pig antisera (1:1000) against RV1B. RV-specific band suspected to be VP1 due to predicted molecular weight of $39 \mathrm{kDa}$ is denoted by arrow. (B) Antisera from naive mice (open symbols) or mice infected with RV1B (closed symbols) were analysed by ELISA for IgG2a and IgG1 binding to purified recombinant RV14 or RV89 VP1. Data are presented as serial dilutions of individual antisera ( $n=2$, naive and RV1B infected).

Interestingly, this immunisation strategy differed from RV infections in that the production of IgG1 that bound strongly to RV89 VP1 was induced (Fig. 6C). Antisera from immunised mice was also analysed for binding to RV preparations by Western blot and was shown to react similarly to sera from $\mathrm{RV}$ infected mice in that mouse IgG bound to a RV-specific $39 \mathrm{kDa}$ protein common to RV1B, RV29 and RV16 preparations (arrow) consistent with VP1 (Fig. 6D). UV treatment of the RV preparations promoted loss of antigenic epitopes by protein cross-linking/aggregation and did not allow recognition by the antisera. We also determined the ability of these sera to neutralise RV in vitro. The antisera neutralised RV1B at an endpoint dilution of 1:640 and also neutralised RV16 at an endpoint dilution of 1:40 whereas sera from mice administered Freund's adjuvant alone contained no neutralising activity (Fig. 6E). RV1B challenge of immunised mice increased the numbers of $\mathrm{CD}^{-} \mathrm{CD} 19^{+} \mathrm{B}$ lymphocytes found in BAL, when compared to challenge of mice receiving adjuvant alone, without altering the numbers of these cells found in lungs (SFig. 1C).

\section{Discussion}

Antibody responses to RV infection are not completely understood and are difficult to study in adults and children due to the fact that humans are infected within months after birth and then
A
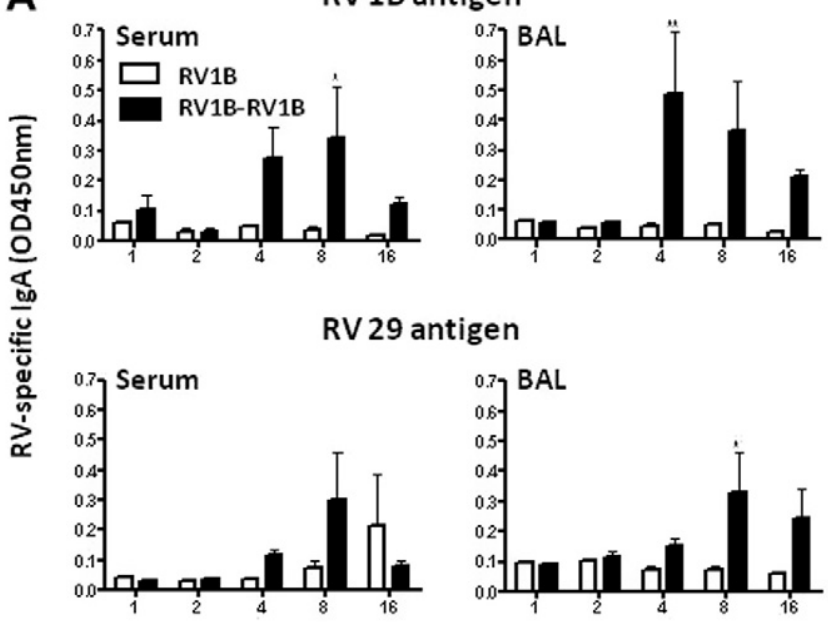

Day after RV infection

\section{B}
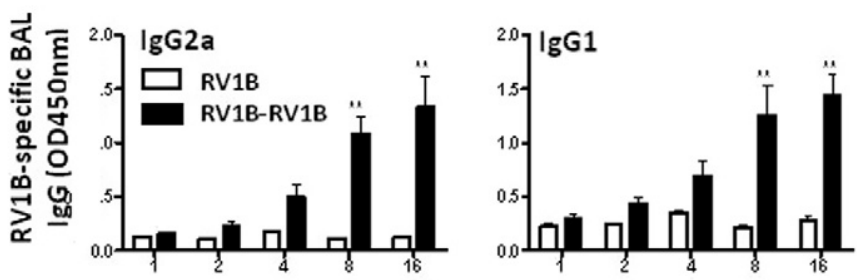

Day after RV infection

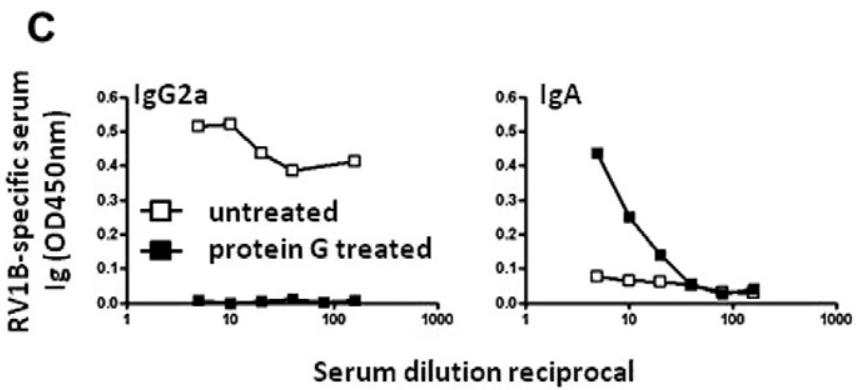

Fig. 3. Induction of RV-specific IgA and mucosal antibody responses requires two RV infections. BALB/c mice were infected with RV1B or mock infected with PBS, left for 30 days then re-infected with RV1B, sacrificed at the indicated time points and serum and BAL obtained. (A) Diluted serum (1:50) or BAL (1:4) from single (white bars) or double (black bars) RV1B infected mice was added to ELISA plates coated with purified RV1B (A, upper panel) or purified RV29 (A, lower panel) and assessed for IgA binding. (B) Diluted BAL (1:4) from single infected (white bars) or double RV1B infected (black bars) mice was also assessed for IgG2a and IgG1 binding to RV1B. Data are presented as the mean \pm SEM $\left({ }^{*} p<0.05,{ }^{* *} p<0.01\right.$, for single RV1B infection vs. double RV1B infections). (C) Serum from BALB/c mice infected five times with RV1B was pooled and allowed to bind with protein G Sepharose to deplete IgG. The unbound fraction (closed squares) was compared with untreated serum (open squares) for levels of IgG2a and IgA binding to RV1B.

continuously throughout life (Turner, 1997). As a result all humans possess high levels of RV-specific antibodies that complicate studies into RV antibody-mediated immunity and vaccine development. To avoid these problems, in this study we have characterised the generation of antibody responses to RV following infection and immunisation of mice. To our knowledge this is the first study to examine antibody responses to RV in vivo in an immunologically naive host. Our results showed that significant cross serotype $\operatorname{IgG}$ responses in serum and BAL are generated in 

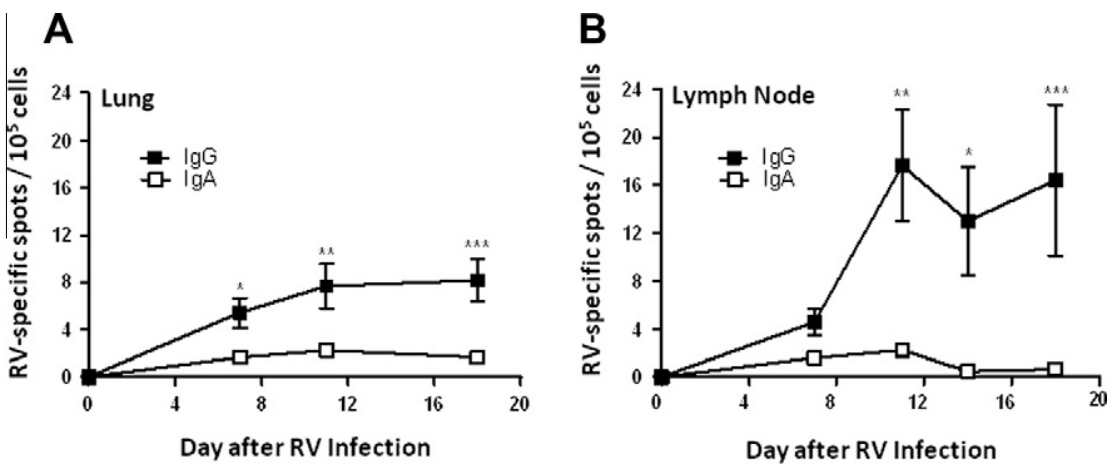

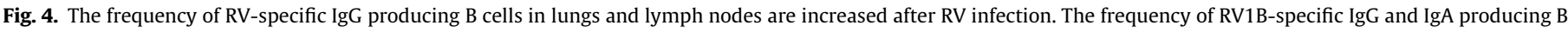

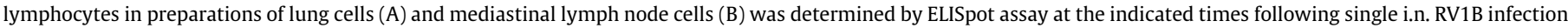
and in naive mice (Day 0). Data are presented as the mean $\pm \operatorname{SEM}\left({ }^{*} p<0.05,{ }^{* *} p<0.01,{ }^{* * *} p<0.001\right.$, for $\operatorname{IgG}$ or $\operatorname{IgA}$ vs. time 0$)$.

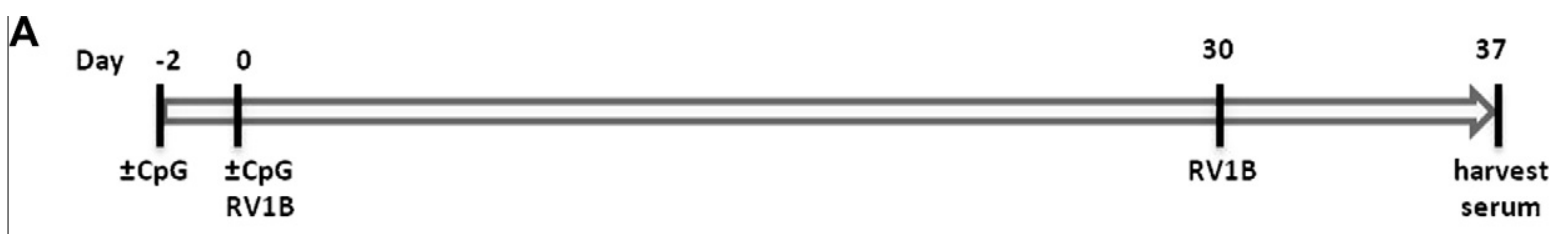

B

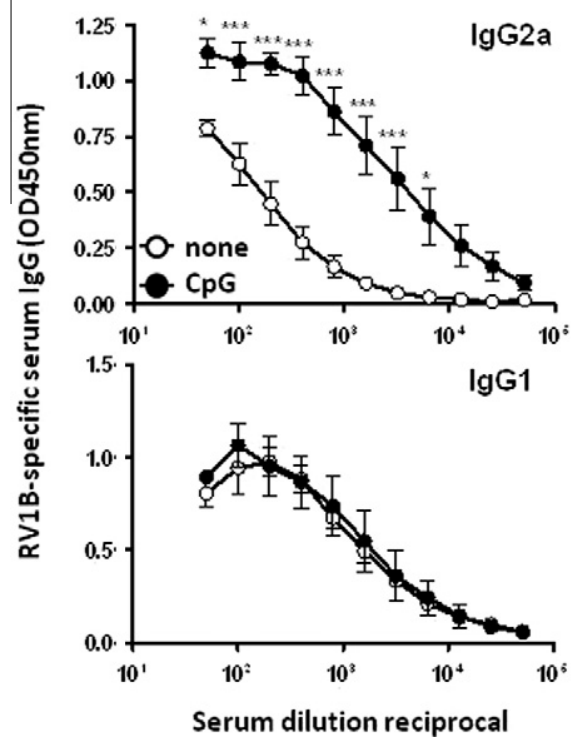

C

\section{RV1B-RV1B CpgRV1B-RV1B}
RV16
n.d.
n.d.

In vitro RV1B and RV16 neutralisation assay serum endpoint (final dilution of serum resulting in complete protection from RV induced $C P E$ ). Mice were infected twice with RV1B with or without prior $\mathrm{CpG}$ administration as shown in $\mathrm{A}$. n.d. $=$ no neutralisation detected.

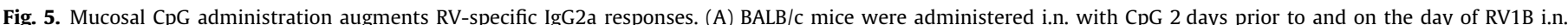

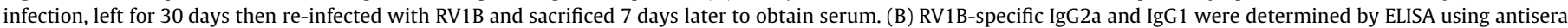

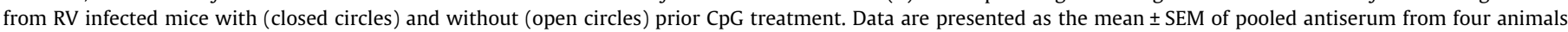

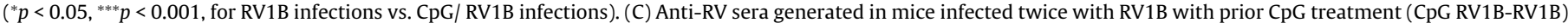

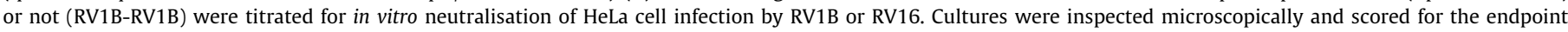
dilution of sera that displayed complete protection from cytopathic effect (CPE).

mice following i.n. RV infection although the production of robust IgA responses and protective neutralising antibodies required repeat or multiple infections. The dominant antibody target for cross-serotype recognition appeared to be the RV capsid protein VP1. The mouse model also allows for investigations of specific antibody responses following adjuvant administration, immunisation and RV challenge demonstrating its utility for the analyses and development of RV vaccine approaches.

RV infections are common throughout life in humans making $\mathrm{RV}$ one of the most successful human viral pathogens. It has been estimated that children can get up to $12 \mathrm{RV}$ infections per year and adults on average 2-5 infections (Turner, 1997). RVs exist as more than 100 serotypes and serological findings demonstrate that protective antibodies generated following infection with one serotype neutralise that serotype but provide little to no cross-protection to other serotypes (Gwaltney, 1985). Because many serotypes of RV are capable of circulating in the population simultaneously and protective neutralising antibody is highly serotype specific, humans are frequently infected by RV serotypes even though they may possess antibodies that are cross-serotype binding. Similarly, as we have shown here with immunologically naive mice, single $\mathrm{RV}$ infections generated cross-serotype binding but non-neutralis- 

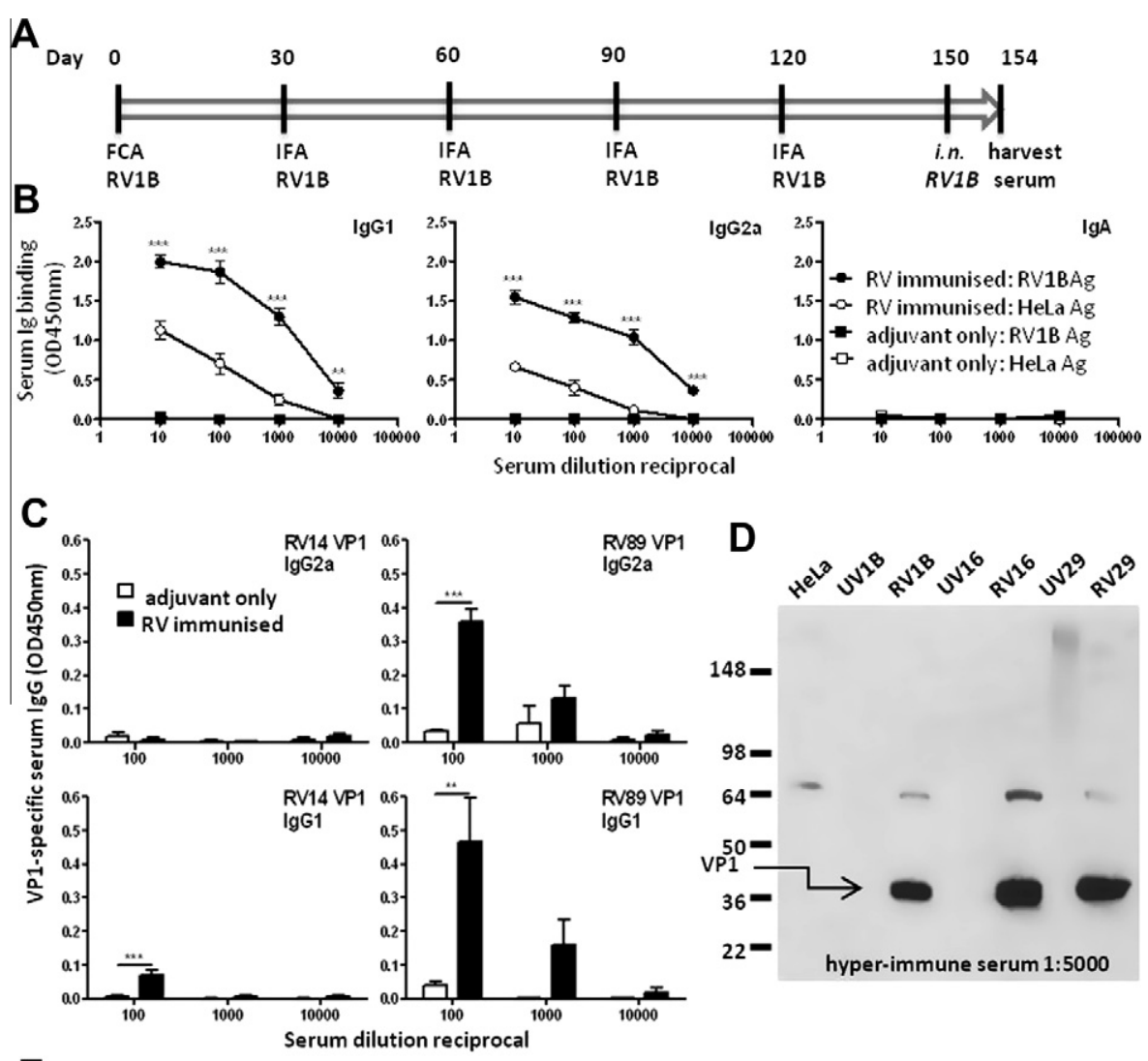

E

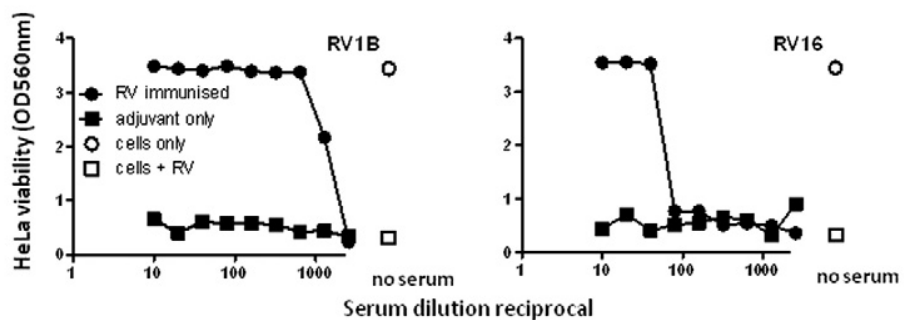

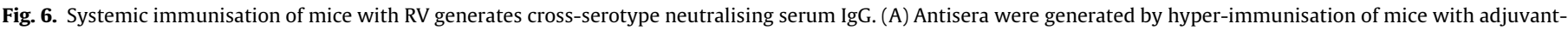

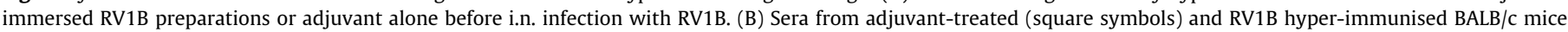

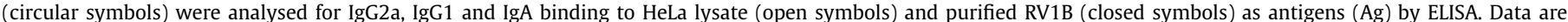

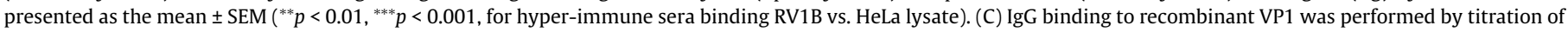

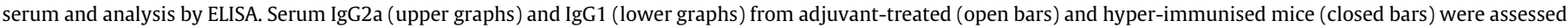

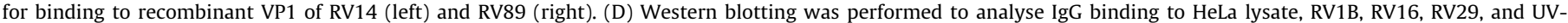

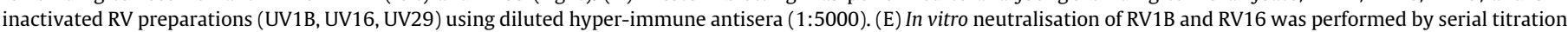

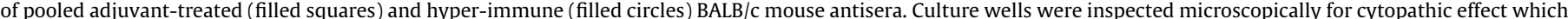

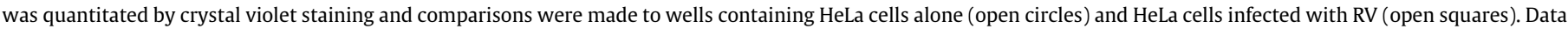
are presented as the mean $\pm \operatorname{SEM}\left({ }^{* *} p<0.01,{ }^{* * *} p<0.001\right.$, for hyper-immune serum vs. adjuvant only).

ing antibodies. To generate higher avidity IgG binding, IgA and neutralising antibody responses, two RV infections, Th1-promoting adjuvants or immunisation with RV preparations in adjuvant were required. This is in contrast to humans where a single infection is often sufficient to generate systemic and mucosal antibody responses capable of serotype-specific neutralisation (Douglas et al., 1967), however since humans are frequently infected with $\mathrm{RV}$, it is likely that pre-existing pools of cross-serotype CD4 helper $\mathrm{T}$ cells generated to these prior RV infections may help prime the response to infection with a new RV serotype. This priming effect is presumably also influenced by age, with young children and infants having been exposed to significantly fewer RV infections and also being susceptible to more frequent RV infections (Taylor-Robinson et al., 1963).

In humans, nasal antibodies of the IgA class have been shown to be critical for protection against RV infection (Barclay and AlNakib, 1987; Barclay et al., 1988) and patients with hypogamma- globulinemia develop persistent RV infections despite adequate intravenous immunoglobulin (IVIg) replacement therapy further highlighting the importance of secretory IgA (Kainulainen et al., 2010). RV-specific secretory IgA is rarely present in the absence of serum IgA and rarely absent when serum titres are high, therefore serum IgA should be a useful marker for the development of mucosal humoral immunity to RV. We identified both mucosal and systemic IgA responses to RV in mice but these required more than one RV infection. These IgA responses were lower than the IgG responses found in both serum and lung washes and are consistent with our detection of a reduced frequency of B cells producing RVspecific IgA compared to IgG in mediastinal lymph nodes and lungs. Systemic immunisation of mice with RV in Freund's adjuvant did not promote IgA responses despite generating high-titre neutralising IgG responses, suggesting that the route of RV administration and virus replication might be critical for optimal IgA responses. However, Barnett et al. have previously demonstrated 
that IgA-producing hybridomas specific to RV are generated after immunisation of mice with adjuvanted RV peptides (Barnett et al., 1995, 1993). Nevertheless, a stronger IgG response in mice may still be biologically significant for RV infection since it is known that secretory IgA protects the upper respiratory tract from influenza but IgG is more important for protection of the lungs (Renegar et al., 2004).

Our results indicate that the RV capsid protein VP1 is a major target of the antibody response in infected mice. We did not detect IgG to VP2 or VP3 despite the fact that we used infectious RV preparations made from HeLa cell cultures in our assays and preparations made in this way have been shown to contain VP1, VP2 and VP3 (Johnston et al., 1998). For our studies, recombinant VP2 and VP3 were not available and the western blot analyses relied on denatured and reduced viral antigen which may have lost potential conformational epitopes within VP2 and VP3. Most antibodies generated to RV infection in humans are directed against epitopes found on surface-exposed structures of VP1, VP2 and VP3 (Carey et al., 1992). Although there is evidence that the $\mathrm{N}$-terminus of VP4 may be transiently exposed at the capsid surface in a process known as capsid breathing (Lewis et al., 1998) and antibodies to this region of VP4 are cross-serotype protective (Katpally et al., 2009), naturally occurring human antibodies to VP4 have not been described. A recent study by Niespodziana et al. revealed that children with RV-induced respiratory symptoms primarily generate $\operatorname{IgG1}$ and $\operatorname{IgA}$ to $\mathrm{N}$-terminal regions of VP1 and that responses to VP2, VP3 and VP4 were significantly lower (Niespodziana et al., 2011). Interestingly the major epitope of these VP1-specific antibodies is located inside the viral capsid and as such is a non-neutralising epitope, suggesting that misdirection of antibody responses in addition to strain variability can explain the escape of RV to protective immunity (Niespodziana et al., 2011). In the current study we have shown by western blotting of denatured RV preparations and by immunoassay of recombinant purified VP1 that mice infected with RV generate IgG that binds to VP1 of multiple RV serotypes. However this binding was sufficient for neutralisation of just the homologous RV serotype suggesting the importance of fine specificity for VP1. Furthermore, the IgG subclass IgG2a was found to be the dominant antibody class interacting with recombinant VP1 even though we demonstrated both IgG2a and IgG1 responses to intact viral preparations. IgG2a has been reported to be commonly produced by mice in response to viral infections (Coutelier et al., 1987) and may be a more effective neutralising antibody due to its ability to fix complement (Neuberger and Rajewsky, 1981). Our data is highly suggestive of IgG2a targeting a linear cross reactive epitope of VP1 following RV infection of mice. Since VP1 is the dominant and most external of the RV capsid proteins and contains receptor binding sites for both the major and minor group RV (Vlasak et al., 2003), it is therefore not surprising that mice infected with RV predominantly generate antibodies to this region of the capsid. VP1 is a useful antibody target as a recent study by Edlmayr et al., (Edlmayr et al., 2011) has revealed that immunisation of rabbits with recombinant VP1 generates antisera with broad cross-serotype neutralising properties. Significantly, there is a high degree of amino acid identity between VP1 within both type A and B RV serotypes and VP1 also contains several conserved domains, the drug binding pocket for pleconaril and two of the four neutralising immunogenic sites targeted by antibodies (Laine et al., 2006; Ledford et al., 2004; Rossmann et al., 1985; Sherry et al., 1986).

It is important to understand the development of antibody responses to RV as young children are obvious vaccine candidates for prevention of early recurrent RV infections which have been reported to be associated with increased risk of asthma development (Jackson et al., 2008) and RV-induced exacerbations of asthma (Murray et al., 2006). In this regard, the study of antibody responses in mice infected or immunised with RV add to the current limited knowledge regarding binding and neutralising antibody responses to RV in humans. We have established the utility of the mouse model by demonstrating the induction of cross-serotype RV-specific antibodies following infection and the use of two different adjuvant approaches to promote neutralising responses. This is the first in vivo study of the priming of antibody responses to RV infection and taken together, these results and our model system have important implications for RV vaccine design. Our studies suggest that RV vaccine approaches need to target the lung using defined highly conserved RV epitopes to generate cross-serotype local mucosal IgA responses which may be more suitable for protection than the systemic IgG responses often favoured. Further studies, such as different strains of mice (e.g. C57Bl/6), the use of mice transgenic for specific antibody genes (e.g. human Ig V regions) or with disrupted IgG genes, other adjuvants, and the transfer of immune serum to assess protection and subsequent analyses in our model system will increase the understanding of cross-protective antibody responses to RV and lead to the generation of a vaccine for this important human respiratory viral pathogen.

\section{Acknowledgements}

This work was supported by Sanofi Pasteur, the Centre for Respiratory Infection of Imperial College London grant \# 083567/ $\mathrm{Z} / 07 / \mathrm{Z}$, the MRC \& Asthma UK Centre in Allergic Mechanisms of Asthma grant \# G1000758, grants FWF-Doctoral Program IAI, Christian Doppler Research Association, and Biomay, Vienna, Austria to RV and by the European Commission's Seventh Framework programme under grant agreement $N^{\circ} 260895$ (PreDicta).

\section{Appendix A. Supplementary data}

Supplementary data associated with this article can be found, in the online version, at http://dx.doi.org/10.1016/j.antiviral.2012. 06.006 .

\section{References}

Barclay, W.S., Al-Nakib, W., 1987. An ELISA for the detection of rhinovirus specific antibody in serum and nasal secretion. J. Virol. Methods 15, 53-64.

Barclay, W.S., Callow, K.A., Sergeant, M., al-Nakib, W., 1988. Evaluation of an enzyme-linked immunosorbent assay that measures rhinovirus-specific antibodies in human sera and nasal secretions. J. Med. Virol. 25, 475-482.

Barclay, W.S., al-Nakib, W., Higgins, P.G., Tyrrell, D.A., 1989. The time course of the humoral immune response to rhinovirus infection. Epidemiol. Infect. 103, 659669.

Barnett, P.V., Rowlands, D.J., Parry, N.R., 1993. Characterization of monoclonal antibodies raised against a synthetic peptide capable of inducing a neutralizing response to human rhinovirus type 2. J. Gen. Virol. 74 (Pt 7), 1295-1302.

Barnett, P.V., Rowlands, D.J., Campbell, R.O., Parry, N.R., 1995. Monoclonal antibodies to a peptide of human rhinovirus type 2 with different specificities recognize the same minimum sequence. J. Gen. Virol. 76 (Pt 5), 1255-1261.

Bartlett, N.W., Walton, R.P., Edwards, M.R., Aniscenko, J., Caramori, G., Zhu, J., Glanville, N., Choy, K.J., Jourdan, P., Burnet, J., Tuthill, T.J., Pedrick, M.S., Hurle, M.J., Plumpton, C., Sharp, N.A., Bussell, J.N., Swallow, D.M., Schwarze, J., Guy, B., Almond, J.W., Jeffery, P.K., Lloyd, C.M., Papi, A., Killington, R.A., Rowlands, D.J., Blair, E.D., Clarke, N.J., Johnston, S.L., 2008. Mouse models of rhinovirus-induced disease and exacerbation of allergic airway inflammation. Nat. Med. 14, 199-204

Carey, B.S., Barclay, W.S., Russell, S.M., Tyrrell, D.A., 1992. The specificity of antibodies induced by infection with rhinovirus type 2. J. Med. Virol. 36, 251-258.

Conant, R.M., Hamparian, V.V., 1968a. Rhinoviruses: Basis for a numbering system. 1 HeLa cells for propagationand serologic procedures. J. Immunol. 100, 107-113.

Conant, R.M., Hamparian, V.V., 1968b. Rhinoviruses: Basis for a numbering system. II. Serologic characterization of prototype strains. J. Immunol. 100, 114-119.

Coutelier, J.P., van der Logt, J.T., Heessen, F.W., Warnier, G., Van Snick, J., 1987. IgG2a restriction of murine antibodies elicited by viral infections. J. Exp. Med. 165, 6469.

Douglas Jr., R.G., Rossen, R.D., Butler, W.T., Couch, R.B., 1967. Rhinovirus neutralizing antibody in tears, parotid saliva, nasal secretions and serum. J. Immunol. 99, 297-303. 
Edlmayr, J., Niespodziana, K., Linhart, B., Focke-Tejkl, M., Westritschnig, K., Scheiblhofer, S., Stoecklinger, A., Kneidinger, M., Valent, P., Campana, R., Thalhamer, J., Popow-Kraupp, T., Valenta, R., 2009. A combination vaccine for allergy and rhinovirus infections based on rhinovirus-derived surface protein VP1 and a nonallergenic peptide of the major timothy grass pollen allergen Phl p 1. J. Immunol. 182, 6298-6306.

Edlmayr, J., Niespodziana, K., Popow-Kraupp, T., Krzyzanek, V., Focke-Tejkl, M., Blaas, D., Grote, M., Valenta, R., 2011. Antibodies induced with recombinant VP1 from human rhinovirus exhibit cross-neutralisation. Eur. Respir. J. 37, 44-52.

Fox, J.P., 1976. Is a rhinovirus vaccine possible? Am. J. Epidemiol. 103, 345-354.

Greenberg, S.B., 2007. Rhinovirus and coronavirus infections. Semin. Respir. Crit. Care Med. 28, 182-192.

Greve, J.M., Davis, G., Meyer, A.M., Forte, C.P., Yost, S.C., Marlor, C.W., Kamarck, M.E. McClelland, A., 1989. The major human rhinovirus receptor is ICAM-1. Cell 56, 839-847.

Gwaltney Jr., J.M., 1985. Virology and immunology of the common cold. Rhinol. 23, 265-271.

Hofer, F., Gruenberger, M., Kowalski, H., Machat, H., Huettinger, M., Kuechler, E., Blaas, D., 1994. Members of the low density lipoprotein receptor family mediate cell entry of a minor-group common cold virus. Proc. Natl. Acad. Sci. USA. 91, 1839-1842.

Jackson, D.J., Gangnon, R.E., Evans, M.D., Roberg, K.A., Anderson, E.L., Pappas, T.E., Printz, M.C., Lee, W.M., Shult, P.A., Reisdorf, E., Carlson-Dakes, K.T., Salazar, L.P. DaSilva, D.F., Tisler, C.J., Gern, J.E., Lemanske Jr., R.F., 2008. Wheezing rhinovirus illnesses in early life predict asthma development in high-risk children. Am. J. Respir. Crit. Care Med. 178, 667-672.

Johnston, S.L., Pattemore, P.K., Sanderson, G., Smith, S., Lampe, F., Josephs, L., Symington, P., O’Toole, S., Myint, S.H., Tyrrell, D.A., et al., 1995. Community study of role of viral infections in exacerbations of asthma in 9-11 year old children. Bmj 310, 1225-1229.

Johnston, S.L., Papi, A., Bates, P.J., Mastronarde, J.G., Monick, M.M., Hunninghake G.W., 1998. Low grade rhinovirus infection induces a prolonged release of IL-8 in pulmonary epithelium. J. Immunol. 160, 6172-6181.

Kainulainen, L., Vuorinen, T., Rantakokko-Jalava, K., Osterback, R., Ruuskanen, O., 2010. Recurrent and persistent respiratory tract viral infections in patients with primary hypogammaglobulinemia. J. Allergy Clin. Immunol. 126, 120-126.

Katpally, U., Fu, T.M., Freed, D.C., Casimiro, D.R., Smith, T.J., 2009. Antibodies to the buried $\mathrm{N}$ terminus of rhinovirus VP4 exhibit cross-serotypic neutralization. J. Virol. 83, 7040-7048.

Knopf, H.L., Perkins, J.C., Bertran, D.M., Kapikian, A.Z., Chanock, R.M., 1970. Analysis of the neutralizing activity in nasal wash and serum following intranasal vaccinaion with inactivated type 13 rhinovirus. J. Immunol. 104, 566-573.

Krieg, A.M., 2002. CpG motifs in bacterial DNA and their immune effects. Annu. Rev. Immunol. 20, 709-760.

Laine, P., Blomqvist, S., Savolainen, C., Andries, K., Hovi, T., 2006. Alignment of capsid protein VP1 sequences of all human rhinovirus prototype strains: conserved motifs and functional domains. J. Gen. Virol. 87, 129-138.
Ledford, R.M., Patel, N.R., Demenczuk, T.M., Watanyar, A., Herbertz, T., Collett, M.S. Pevear, D.C., 2004. VP1 sequencing of all human rhinovirus serotypes: insights into genus phylogeny and susceptibility to antiviral capsid-binding compounds. J. Virol. 78, 3663-3674.

Lewis, J.K., Bothner, B., Smith, T.J., Siuzdak, G., 1998. Antiviral agent blocks breathing of the common cold virus. Proc. Natl. Acad. Sci. USA. 95, 6774-6778.

Murray, C.S., Poletti, G., Kebadze, T., Morris, J., Woodcock, A., Johnston, S.L., Custovic, A., 2006. Study of modifiable risk factors for asthma exacerbations: virus infection and allergen exposure increase the risk of asthma hospital admissions in children. Thorax 61, 376-382.

Neuberger, M.S., Rajewsky, K., 1981. Activation of mouse complement by monoclonal mouse antibodies. Eur. J. Immunol. 11, 1012-1016.

Niespodziana, K., Napora, K., Cabauatan, C., Focke-Tejkl, M., Keller, W., Niederberger, V., Tsolia, M., Christodoulou, I., Papadopoulos, N.G., Valenta, R., 2011. Misdirected antibody responses against an N-terminal epitope on human rhinovirus VP1 as explanation for recurrent RV infections. Faseb. J. 25, 25.

Perkins, J.C., Tucker, D.N., Knope, H.L., Wenzel, R.P., Hornick, R.B., Kapikian, A.Z., Chanock, R.M., 1969. Evidence for protective effect of an inactivated rhinovirus vaccine administered by the nasal route. Am. J. Epidemiol. 90, 319-326.

Renegar, K.B., Small Jr., P.A., Boykins, L.G., Wright, P.F., 2004. Role of IgA versus IgG in the control of influenza viral infection in the murine respiratory tract. J. Immunol. 173, 1978-1986.

Rhinoviruses: a numbering system., 1967. Nature 213, 761-762.

Rossmann, M.G., Arnold, E., Erickson, J.W., Frankenberger, E.A., Griffith, J.P., Hecht, H.J., Johnson, J.E., Kamer, G., Luo, M., Mosser, A.G., et al., 1985. Structure of a human common cold virus and functional relationship to other picornaviruses. Nature 317, 145-153.

Schieble, J.H., Fox, V.L., Lester, F., Lennette, E.H., 1974. Rhinoviruses: an antigenic study of the prototype virus strains. Proc. Soc. Exp. Biol. Med. 147, 541-545.

Sherry, B., Mosser, A.G., Colonno, R.J., Rueckert, R.R., 1986. Use of monoclonal antibodies to identify four neutralization immunogens on a common cold picornavirus, human rhinovirus 14. J. Virol. 57, 246-257.

Skern, T. Neubauer, C., Frasel, L, Grundler, P., Sommergruber, W., Zorn, M. Kuechler, E., Blaas, D., 1987. A neutralizing epitope on human rhinovirus type 2 includes amino acid residues between 153 and 164 of virus capsid protein VP2. J. Gen. Virol. 68 (Pt 2), 315-323.

Taylor-Robinson, D., Johnson, K.M., Bloom, H.H., Parrott, R.H., Mufson, M.A., Chanock, R.M., 1963. Rhinovirus neutralizing antibody responses and their measurement. Am. J. Hyg. 78, 285-292.

Turner, R.B., 1997. Epidemiology, pathogenesis, and treatment of the common cold. Ann Allergy Asthma Immunol. 78, 531-539; quiz 539-540.

Vlasak, M., Blomqvist, S., Hovi, T., Hewat, E., Blaas, D., 2003. Sequence and structure of human rhinoviruses reveal the basis of receptor discrimination. J. Virol. 77, 6923-6930. 